\title{
Warm Debris Disks with WISE and HST
}

\author{
Deborah Padgett ${ }^{1}$ and Karl Stapelfeldt ${ }^{2}$ \\ ${ }^{1}$ Code 665, NASA Goddard Space Flight Center \\ email: deborah.1.padgett@nasa.gov \\ ${ }^{2}$ Code 667, NASA Goddard Space Flight Center \\ email: karl.r.stapelfeldt@nasa.gov
}

\begin{abstract}
Using $22 \mu \mathrm{m}$ data from the Wide Field Infrared Survey Explorer (WISE), we have completed a sensitive all-sky survey for debris disks in Hipparcos and Tycho catalog stars within $120 \mathrm{pc}$. This warm excess emission traces material in the circumstellar region likely to host terrestrial planets. Several hundred previously unknown debris disk candidates were identified. We are currently performing follow-up observations to characterize the stars, companions, and circumstellar material in these systems with a variety of facilities including Keck, Herschel, and HST. Thirteen WISE debris disks have been observed to date using HST/STIS coronagraphy. Five of these disks have been detected in scattered light. One is a large and highly asymmetric edge-on disk which appears to be both warped and bifurcated.
\end{abstract}

Keywords. stars: circumstellar matter, stars: planetary systems, protoplanetary disks

\section{Introduction}

Circumstellar disks are the most visible signs of other solar systems. Planets and planetesimals (asteroids, comets) begin to form shortly after a star is born. The primordial gas and dust disk that gives birth to the star dissipates in 5-10 Myr due to accretion, giant planet formation, jets, and photoevaporation. Subsequently, a new "debris disk" emerges, consisting of dust grains generated by the collisions of the planetesimals and the evaporation of comets. These grains are quickly blown away by radiation pressure and stellar winds, so to exist for any significant period of time the disk must be continually replenished by the destruction of planetesimals. This is the case for the debris disk around our own Sun, the zodiacal cloud + Kuiper dust belt, formed by collisions in belts of small bodies. The presence of a debris disk therefore indicates the presence of some sort of planetary system.

Debris disks have become especially interesting to the extrasolar planet community because they can betray the presence of planets that would otherwise go unseen. Gravitational interactions between planets and dust can carve out localized gaps, create clearings (rings) within the disk, or generate warps. In our own zodiacal cloud, for instance, Earth has cleared out a region around it through resonant tidal interactions (Dermott et al. 1994). The A star Fomalhaut possesses a debris ring (Kalas et al. 2005) with 133 AU inner radius, 25 AU width, sharp inner edge, and center that is offset 15 AU from the stellar position - certain signs of a planetary mass companion sculpting the disk. A second, even more eccentric ring has been found around the solar-type star HD 202628 (Krist et al. 2012). Dynamical modeling of disks has matured to the point where structures can be related to the likely mass and orbital characteristics of the perturbing object (Quillen 2006, Chiang et al. 2009, Rodigas et al. 2014). In the Fomalhaut case, HST imaging Kalas et al. (2008) identified an unusual object orbiting near the ring inner edge (Kalas et al. 2013). The four planets imaged in the HR 8799 system (Marois et al. 2008, Marois 
et al. 2010) were subsequently shown to be orbiting in a gap between two debris belts ( $\mathrm{Su}$ et al. 2009). The planet responsible for the inner disk warp in $\beta$ Pictoris was finally found by Lagrange et al. (2010). HD $95086 \mathrm{~b}$ was also imaged in a debris disk system (Rameau et al. 2013). It is very significant that many of the systems with directly imaged exoplanets also host bright debris disks. Mapping the structure of debris disks can thus point the way toward direct imaging of their perturbing planets.

In the 1980s IRAS provided the first evidence of debris disks with measurements of excess thermal emission around stars like Vega, Fomalhaut, Beta Pictoris and Epsilon Eridani. It was followed by ISO, Spitzer, Akari, and Herschel, which have added hundreds more potential debris disk systems to the inventory. However, as pointed observatories, ISO, Spitzer, and Herschel were able to survey only a fraction of nearby stars for infrared excess, so they cannot be expected to have identified all the brightest disks in the solar neighborhood. For solar-type stars, disks with excess emission at $22 \mu \mathrm{m}$ occur primarily around young stars as important signposts of planetary system evolution (Rieke et al. 2005, Carpenter et al. 2009). The youth of such systems means they are excellent potential homes for bright young planets that could be directly imaged using high contrast techniques, which might also reveal disk structure unresolved at the low resolution currently available for most thermal IR imagery.

\section{WISE Debris Disk Candidates}

In 2010, the Wide-field Infrared Survey Explorer (WISE; Wright et al. 2010) performed an all-sky search for debris disks in the mid-infrared. With $22 \mu \mathrm{m}$ sensitivity more than 100 times that of IRAS at $25 \mu \mathrm{m}$, WISE is capable of finding warm debris disks around $\mathrm{G}$ stars within $100 \mathrm{pc}$. With our knowledge of the WISE catalog and data products, we merged the all-sky cryogenic WISE catalog with the Hipparcos catalog and found over 566 stars with greater than $4 \sigma$ mid-IR excess (from either [3.4]-[22] or [12]-[22]) within $120 \mathrm{pc}$, of which 350 were not known to have debris disks in the pre-WISE era (McDonald et al. 2012, Patel et al. 2014, etc.). Merging the Tycho-2 catalog with WISE yielded more than 450 additional debris disk candidates with proper motions consistent with distances less than 120 pc (Padgett et al. (2015a), in preparation). The number of $4 \sigma$ excess detections decreased for the newer ALLWISE data release, as the uncertainties for saturated source photometry for [3.4] (W1) and [4.5] (W2) magnitudes increased with the addition of warm WISE data for those bands. These are high-quality detections with position matches between Hipparcos and WISE better than 0.3 arcsec, smoothly continuous photometry with 2MASS measurements, and individually vetted by visual checks of the WISE and 2MASS images. Ten percent of these sources show WISE colors consistent with $12 \mu \mathrm{m}$ excess as well as $22 \mu \mathrm{m}$ excess. Half of the WISE/HIP candidates have radial velocities, and known members of young associations are well represented. Some previously unstudied sources are apparently comoving with known associations but are at large spatial separations (Padgett et al. 2015a, in preparation).

The WISE debris disk candidate stars are in the process of being characterized at a variety of wavelengths. About 120 solar-type and A stars from this sample were observed at $70 \mu \mathrm{m}$ and $160 \mu \mathrm{m}$ by Herschel/PACS. Nearly all of the sources were detected at these wavelengths, although about $20 \%$ show enough of an offset between the stellar position and the long wavelength emission to raise suspicion of chance alignment between the star and a background star-forming galaxy. This level of contamination is not surprising for the Herschel sample since 98 of the targets are solar-type stars. Of the stars with convincing far-IR excess, the derived dust temperatures range from $60-150 \mathrm{~K}$ with roughly equal numbers below and above $90 \mathrm{~K}$. A significant number have evidence for at least two temperature components in their spectral energy distributions (Padgett 

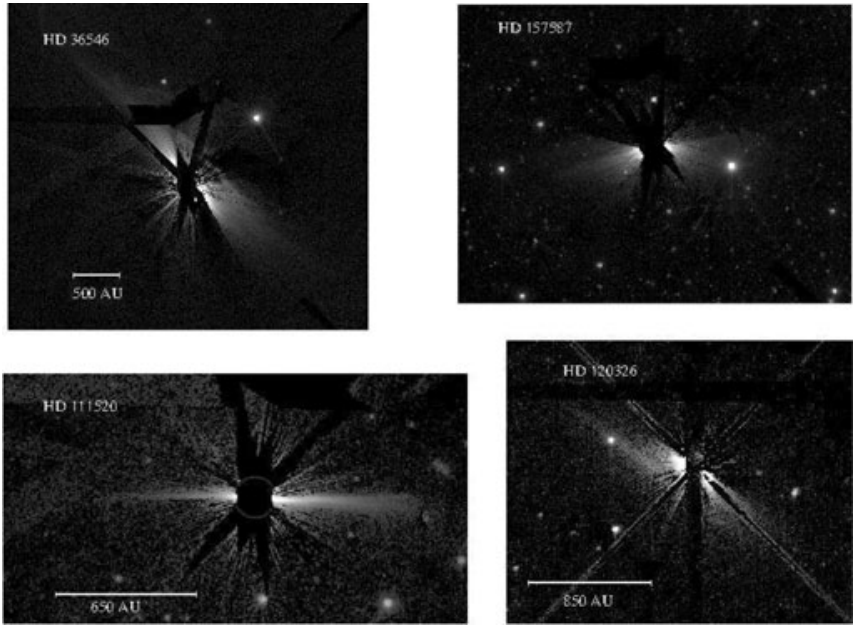

Figure 1. STIS coronagraph images of WISE-discovered warm debris disks.

et al. 2015b, in preparation). The WISE debris disk candidates are also the subject of an ongoing Keck near-IR coronographic survey for substellar companions (Hinkley et al. 2014).

\section{Hubble Space Telescope Coronography of WISE Disks}

Debris disks are difficult to see in scattered light, as even the brightest have total optical fluxes of only a fraction of a percent of their stars'. The first debris disk ever imaged was $\beta$ Pictoris (Smith \& Terrile 1984), which was observed using a coronagraph to reduce the glare from the star. After many fruitless years of searching from the ground, other debris disks were finally resolved at millimeter, infrared, and optical wavelengths beginning in the late 1990s: HR 4796, Vega, Fomalhaut, $\epsilon$ Eridani, and HD 141569A; see Wyatt (2008) for a review. During the past decade the number of resolved debris disks has nearly quadrupled. However, only about half of these are detected in scattered light where 0.1 arcsec resolution imaging is available (see the compilation at http://circumstellardisks.org). Some large disks have also been resolved in far-infrared thermal emission by Herschel (Matthews et al. 2010, Lohne et al. 2012, Acke et al. 2012, Booth et al. 2013). Many of the brightest disks have been imaged using adaptive optics coronagraphy. Yet the disks imaged thus far represent spectral types A through M with ages ranging from a few million to several billion years: too sparse a sample from which to derive any correlations that could reveal the physics behind the evolution of these systems.

The optical depths of debris disks (which determine their scattered light brightnesses) are proportional to the strength of their infrared excess. Disks with fractional infrared luminosities $L_{\text {dust }} / L_{\text {star }}>10^{-4}$ have been shown to be detectable in scattered light by HST. Not all such disks have been detected, however, as some may be smaller than the inner working angle of the coronagraph, have low albedos or have face-on orientations that make detection more difficult. In HST cycles 19 and 20, we observed a total of 13 WISE debris disk candidates using the STIS coronagraphic wedge. These were selected for high $L_{\text {dust }} / L_{\text {star }}$, proximity, and brightness of excess at WISE $22 \mu \mathrm{m}$ and/or Herschel $70 \mu \mathrm{m}$. They were each observed for two orbits at different roll angles, using self-subtraction to mitigate the remaining scattered light from the point spread function (PSF). Six of the targets showed no scattered light beyond the PSF residuals. Two were resolved as 
star-galaxy pairs, demonstrating the continuing contamination issue posed by superposition of bright stars with the extragalactic background. Five have significant scattered light consistent with the presence of resolved dust structures around the star. Fig. 1 shows the roll-subtracted STIS coronagraph images for four of these objects. One spectacular example is an asymmetric edge-on system $500 \mathrm{AU}$ in radius around HD 111520, an F star in Lower Centarus-Crux (Fig. 1 lower left). The disk appears warped and is five times brighter on the NW side than on the SE. The NW side is also clearly bifurcated, with two distinctly inclined planes (Padgett et al. 2015c, in preparation). We are in the process of characterizing these disks at NIR wavelengths using MagAO (Rodigas et al. 2015, in preparation).

\section{References}

Acke, B., Min, M., Dominik, C., Vandenbussche, B., Sibthorpe, B., Waelkens, C., Olofsson, G., Degroote, P., Smolders, K., \& Pantin, E., et al. 2012, A\& A, 540, 125

Booth, M., Kennedy, G., Sibthorpe, B., Matthews, B. C., Wyatt, M. C., Duchene, G., Kavelaars, J. J., Rodriguez, D., Greave, J. S., Koning, A. et al. 2013, MNRAS, 428, 1263

Carpenter, J. M., Mamajek, E. E., Hillenbrand, L. A., \& Meyer, M. R. 2009, ApJ, 705, 1646

Chiang, E., Kite, E., Graham, J. R., \& Clampin, M. 2009, ApJ, 693, 734

Dermott, S. F., Jayaraman, S., Xu, Y. L., Gustarfson, B. A. S.., \& Liou, J. C. 1994, Nature, 369,719

Hinkley, S., Mawet, D., Stapelfeldt, K., Padgett, D., Morales, F., \& Serabyn, E. 2014, Proceedings of the conference Thirty years of beta Pic and debris disks studies, A.-M. Lagrange \& A. Boccaletti, eds., p.40

Kalas, P., Graham, J. R., \& Clampin, M. 2005, Nature, 435, 1067

Kalas, P., Graham, J., Chiang, E., Fitzgerald, M., Clampin, M., Kite, E., Stapelfeldt, K., Marois, C., \& Krist, J. 2008, Science, 322, 1345

Kalas, P., Graham, J. R., Fitzgerald, M. P., \& Clampin, M. 2013, ApJ, 775, 56

Krist, J. E., Stapelfeldt, K. R., Bryden, G., \& Plavchan, P. 2012, AJ, 144, 45

Lagrange, A.-M., Bonnefoy, M., Chauvin, G., Apai, D., Ehrenreich, D., Boccaletti, A., Gratadour, D., Rouan, D., Mouillet, D., Lacour, S., \& Kaspar, M. 2010, Science, 329, 57

Lohne, T., Eiroa, C., Augereau, J.-C., Ertel, S., Marshall, J. P., Mora, A., Absil, O., Stapelfeldt, K., Thebault, P., Bayo, A., et al. 2012, Astronomische Nachrichten, 333, 441

Marois, C., Macintosh, B., Barman, T., Zuckerman, B., Song, I., Patience, J., Lafreniere, D., \& Doyon, R. 2008, Science, 322, 1348

Marois, C., Zuckerman, B., Konopacky, Q. M., Macintosh, B., \& Barman, T. 2010, Nature, 468, 1080

Matthews, B. C., Sibthorpe, B., Kennedy, G., Phillips, N., Churcher, L., Duchene, G., Greaves, J. S., Lestrade, J.-F., Moro-Martin, A., \& Wyatt, M. C., et al. 2010, A\&AA, 518, 135

McDonald, I., Zijlstra, A., \& Boyer, M. 2012, MNRAS, 427, 343

Patel, R., Metchev, S., \& Heinze, A. 2014, ApJS, 212, 10

Quillen, A. C. 2006, MNRAS, 372, 14

Rameau, J., Chauvin, G., Lagrange, A.-M., Meshkat, T., Boccaletti, A., Quanz, S. P., Currie, T., Mawet, D., Girard, J. H., Bonnefoy, M., \& Kenworthy, M. 2013, ApJ, 779, 26

Rieke, G. H., Su, K. Y. L.., Stansberry, J. A., Trilling, D., Bryden, G., Muzerolle, J., White, B., Gorlova, N., Young, E. T., Beichman, C. A., Stapelfeldt, K. R., \& Hines, D. C. 2005, ApJ, 620,1010

Rodigas, T. J., Malhotra, R., \& Hinz, P. M. 2014, ApJ, 780, 65

Smith, B. \& Terrile, R. 1984, Science, 226, 1421

Su, K. Y. L., Rieke, G. H., Stapelfeldt, K. R., Malhotra, R., Bryden, G., Smith, P. S., Misselt, K. A., Moro-Martin, A., \& Williams, J. P. 2009, ApJ, 705, 314

Wright, E. L., Eisenhardt, P. R. M.., Mainzer, A. K., Ressler, M. E., Cutri, R. M., Jarrett, T., Kirkpatrick, J. D., Padgett, D., McMillan, R. S., Skrutskie, M. et al. 2010, AJ, 140, 1868

Wyatt, M. 2008, ARAA, 46, 339 